\title{
Inoperable Disease
}

National Cancer Institute

\section{Source}

National Cancer Institute. Inoperable Disease. NCI Thesaurus. Code C143015.

Disease state in which the surgical removal of a tumor is not feasible or useful. 